\title{
Pricing Access to the Internet with Partial Information
}

\author{
Ilaria Brunetti ${ }^{1}$, Eitan Altman ${ }^{1}$ and Majed Haddad ${ }^{2}$ \\ ${ }^{1}$ INRIA, Sophia-Antipolis, France \\ ${ }^{2}$ LIA, University of Avignon, France \\ email: \{ilaria.brunetti, eitan.altman, majed.haddad\}@inria.fr
}

\begin{abstract}
We shall consider two competition problems between service providers with asymmetric information. The utility of each one of them depends on the demand it gets and in its price. The demand itself is also a function of the prices of the providers. In both problems there is one provider (super player or player 1) that has more information than the other (player 2) on the demand function. The more informed provider plays first, and then the second observes the move of the first provider and chooses accordingly its own action: it determines its price per unit demand. In the first problem that we consider, the first provider does not control its price (it has a fixed price known to the other provider which does not depend on the information that is unknown to provider 2). Before player 2 takes its action it receives a signal (or a recommendation) from the more informed player, i.e. from provider 1 . The pure actions of provider 1 are thus the possible choices of what signal to send. The second problem that we consider is the same as the first one except that the actions of provider 1 is to choose its price. Since player 2 observes the choice of price of player 1 before it takes its own pricing decision, we can consider the choice of price by player 1 has also a role of signalling. We reduce each one of the problem to an equivalent four by four matrix game.
\end{abstract}

\section{INTRODUCTION}

This note uses Bayesian game theory in order to study pricing issues in competitive environment. We model two problems related to pricing under the assumption that only one player fully knows the demand function. In the first part, we study the signalling problem in which the informed player can signal information to the other one and has to decide on a signalling rule such that, given an optimal reaction of the other player who reacts to the signal, the utility of the informed player would be maximized. In a second scenario that we study, each of the two players determine their prices. Yet the order of the decisions is such that the player that is not informed of the demand function that is used, determines its own price after observing the choice of the first player. We formulate both games as Bayesian games and show how to transform them into equivalent matrix games.

Our goal in this paper is to understand a very simple and tractable model of a two player game with one agent being a content provider more informed than the other. In Section II, we present the signalling game model. We first propose in Section III the signalling game framework where the price of player 1 is fixed and known. We derive utilities for both providers and transform the proposed Bayesian game into a matrix game by considering the demand function as a simple, linear, decreasing function of the provider's price. We then extend in Section III the results to the case where the first provider can choose one of two values as its own price instead of just a single based on a knowledge about the demand. We numerically examine the structure of the utilities in the both cases and study the equilibria in Section V. The paper concludes with a discussion in Section VI.

\section{The Signalling Game Model}

Our starting point is to define two service providers. The demand $d_{i}$ that provider $i$ receives is a function of both its own price as well as of its competitor. We thus write it as $d_{i}\left(p_{1}, p_{2}\right)$. We assume that the utility for provider $i$ is given by

$$
U^{i}=d_{i} p_{i}, \quad i=1,2
$$

Each provider wishes to maximize the expectation of its own utility.

Let us first consider the following scenario, which is common knowledge for both providers (which we call players).

- The price of player 1 is fixed and known.

- Player 2 can choose one of two possible levels of pricing: $\zeta_{l}$ and $\zeta_{h}$.

- The demand $d_{i}$ for each provider is a function of the prices of both providers. The demand function of player 2 is known to both players.

- We assume that the demand of player 1 depends on some parameter $\theta$. $\theta$ is known to player 1 but not to player 2 . It can take one of two values: $\theta_{l}$ and $\theta_{h}$.

The game is then played as follows.

- The value of $\theta$ is chosen by nature according to some distribution $\pi$. It equals $\theta_{r}$ with probability $\pi_{r}$.

- Player 1 observes $\theta$ and then sends a signal to player 2 . It has four pure strategies for signalling: $q_{1} q_{1}, q_{1} q_{2}, q_{2} q_{1}$ and $q_{2} q_{2}$. Here, $q_{i} q_{j}$ is the strategy according to which player 1 signals $q_{i}$ if it observes $\theta_{l}$ and signals $q_{j}$ if it observes $\theta_{h}$.

- Player 2 has also four pure strategies. We write them as $\zeta_{l} \zeta_{l}, \zeta_{l} \zeta_{h}, \zeta_{h} \zeta_{l}$ and $\zeta_{h} \zeta_{h}$. Here, a strategy of the form 
$\zeta_{i} \zeta_{j}$ means choosing $\zeta_{i}$ if player 2 observes a signal $q_{1}$ from player 1 and choosing $\zeta_{j}$ otherwise.

The game that we study is known as a game with "cheap talk"; this is a signaling game where the sender incurs no cost for his signals [1, Sec. 7].

\section{The FIXED PRICE GAME}

Let us first consider the case in which the price $p_{1}$ of player 1 is fixed and known.

We can now represent the game as a $4 \times 4$ matrix game.

Fix $i \in(1,2), j \in(1,2), m \in(l, h), n \in(l, h)$. Assume that player 1 chooses $q_{i} q_{j}$ and that player 2 chooses $\zeta_{m} \zeta_{n}$.

For $r=1,2$, define $\xi(r, i, j)$ to be $i$ if $r=1$ and $j$ otherwise. Player 1 sends signal $q_{\xi(r, i, j)}$ to player 2 if $\theta=\theta_{r}$.

Define $\phi(r, i, j, m, n)$ to be the price chosen by player 2 as a result of the signal $\xi(r, i, j)$ when it uses the strategy $\zeta_{m} \zeta_{n}$. $\phi(r, i, j, m, n)$ equals $\zeta_{m}$ if $\xi(r, i, j)=1$ and is otherwise $\zeta_{n}$.

Then

$$
\begin{aligned}
U^{1}\left(q_{i} q_{j} ; \zeta_{m} \zeta_{n}\right) & =\mathbb{E}_{q_{i} q_{j} ; \zeta_{m} \zeta_{n}}\left[p_{1} d_{1}\left(p_{1}, p_{2}\right)\right] \\
& =\sum_{r=1,2} \pi_{r} \mathbb{E}_{q_{i} q_{j} ; \zeta_{m} \zeta_{n}}\left[p_{1} d_{1}\left(p_{1}, p_{2}\right) \mid \theta=\theta_{r}\right]
\end{aligned}
$$

where $\quad \mathbb{E}_{q_{i} q_{j} ; \zeta_{m} \zeta_{n}}\left[p_{1} d_{1}\left(p_{1}, p_{2}\right) \mid \theta=\theta_{r}\right]=$ $p_{1} d_{1}\left(p_{1}, \phi(r, i, j, m, n)\right)$.

Thus we can rewrite:

$$
U^{1}\left(q_{i} q_{j} ; \zeta_{m} \zeta_{n}\right)=p_{1} \sum_{r=1,2} \pi_{r} d_{1}\left(p_{1}, \phi(r, i, j, m, n)\right)
$$

For player 2 we obtain:

$$
\begin{aligned}
U^{2}\left(q_{i} q_{j} ; \zeta_{m} \zeta_{n}\right) & =\mathbb{E}_{q_{i} q_{j} ; \zeta_{m} \zeta_{n}}\left[p_{2} d_{2}\left(p_{1}, p_{2}\right)\right] \\
& =\sum_{r=1,2} \pi_{r} \cdot \phi(r, i, j, m, n) \cdot d_{2}\left(p_{1}, \phi(r, i, j, m, n)\right.
\end{aligned}
$$

$$
\begin{array}{r}
U^{2}\left(q_{i} q_{j} ; \zeta_{m} \zeta_{n}\right)=\sum_{r=1,2} \pi_{r} \phi(r, i, j, m, n)\left[d_{2}^{0}\left(\theta_{r}\right)-\right. \\
\left.A_{22} \phi(r, i, j, m, n)+A_{12} q_{\xi(r, i, j)}\right]_{+}
\end{array}
$$

\section{NumericAl Results}

Figure 1 and 2 depict the expected utility of both ISPs computed following the procedure outlined in Section II to evaluate the impact of the first ISP (the one who plays first) price on the strategies of both ISPs with parameters $\mathbf{d}_{\mathbf{2}}^{\mathbf{0}}=\left[\theta_{l} \theta_{h}\right]$ and demand matrix $A=\left(\begin{array}{ll}1 & 1 \\ 1 & 1\end{array}\right)$. In the following, we refer to the model in Section III by the fixed price model and the model in Section IV by the pricing model.

where $[x]_{+}=\max \{x, 0\}$ is the positive part of $x$. Assun that (i) $\mathbf{A}$ is known to both players, (ii) $d_{1}^{0}$ is known (as the first player is supposed to know the demand) and (iii) $d_{2}^{0}$ is unknown. It can be written as a function of a parameter $\theta$, i.e., $d_{2}^{0}=d_{2}^{0}(\theta)$.

It follows from the above considerations that the utility functions of player 1 and player 2 can be expressed as:

$$
\begin{gathered}
U^{1}\left(q_{i} q_{j} ; \zeta_{m} \zeta_{n}\right)=p_{1} \sum_{r=1,2} \pi_{r}\left[\theta_{r}-A_{11} p_{1}+A_{21} \phi(r, i, j, m, n)\right] \\
U^{2}\left(q_{i} q_{j} ; \zeta_{m} \zeta_{n}\right)=\sum_{r=1,2} \pi_{r} \cdot \phi(r, i, j, m, n)\left[d_{2}^{0}\left(\theta_{r}\right)-\right. \\
\left.A_{22} \phi(r, i, j, m, n)+A_{12} p_{1}\right]_{+}
\end{gathered}
$$

We first see that, for the fixed price model, the first player's utility is maximized for $p_{1}=15$ while the second player's utility increases linearly as function of $p_{1}$. In particular, we observe a Braess type paradox [2] as the first player experiences the same expected utility for a low and higher price. Interestingly, it is also shown in both figures that the second player performs better when it observes a signal on the strategy chosen by the first player. On the contrary, the first player sees its utility degraded by choosing its price based on the demand level. This suggests that when the first player signals its price to the second player, this latter exploits this information to enhance its expected utility by choosing a lower price resulting in decreasing the first player's utility. 


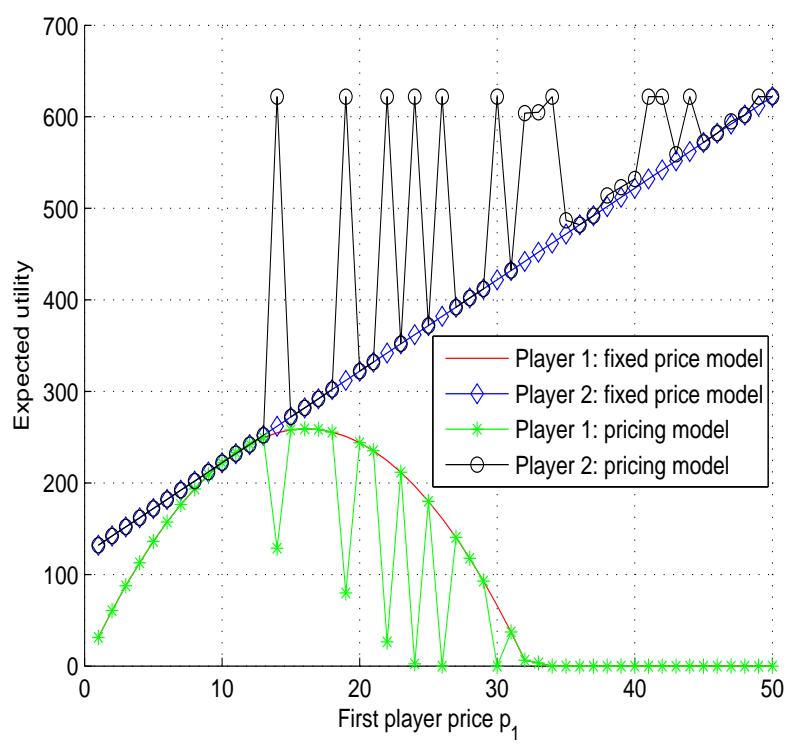

Fig. 1. Service providers' expected utility as function of the first player's price $p_{1}$ with $q_{l}=1, q_{h}=50, \zeta_{l}=1, \zeta_{h}=10, \theta_{l}=22, \theta_{h}=24$.

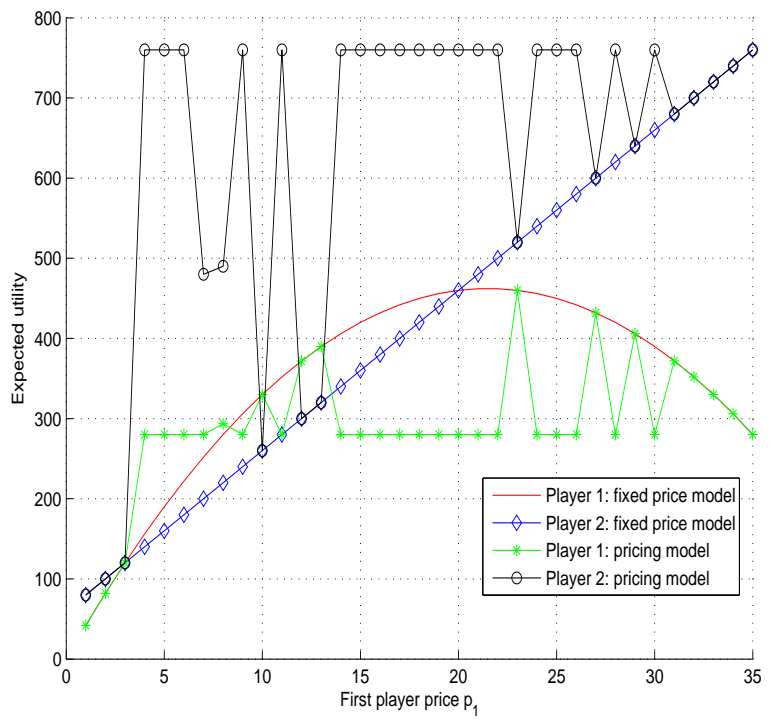

Fig. 2. Service providers' expected utility as function of the first player's price $p_{1}$ with $q_{l}=1, q_{h}=35, \zeta_{l}=1, \zeta_{h}=20, \theta_{l}=22$ and $\theta_{h}=24$.

In Figure 3, we plot service providers' expected utilities as function of the probability $\pi_{h}$ to have a high demand $\theta_{h}$ with $q_{l}=1, q_{h}=20, \zeta_{l}=0, \zeta_{h}=10, \theta_{l}=1$ and $\theta_{h}=20$. As expected, the utility increases linearly as $\pi_{h}$ increases except for the case of first player pricing model. Indeed, as the first player chooses its own price this tends to decrease its utility with respect to the case where the first player has a fixed price. This can be explained by the same arguments in the previous figures.

The mixed equilibria of both players for the pricing model

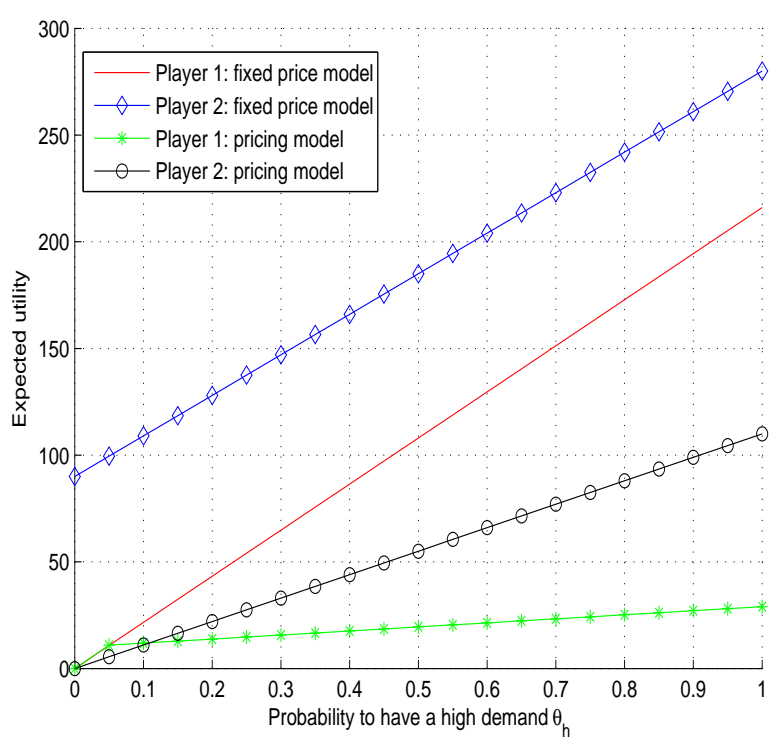

Fig. 3. Service providers' expected utility as function of $\pi_{h}$ with $q_{l}=1, q_{h}$ $=35, \zeta_{l}=0, \zeta_{h}=10, \theta_{l}=1$ and $\theta_{h}=20$.

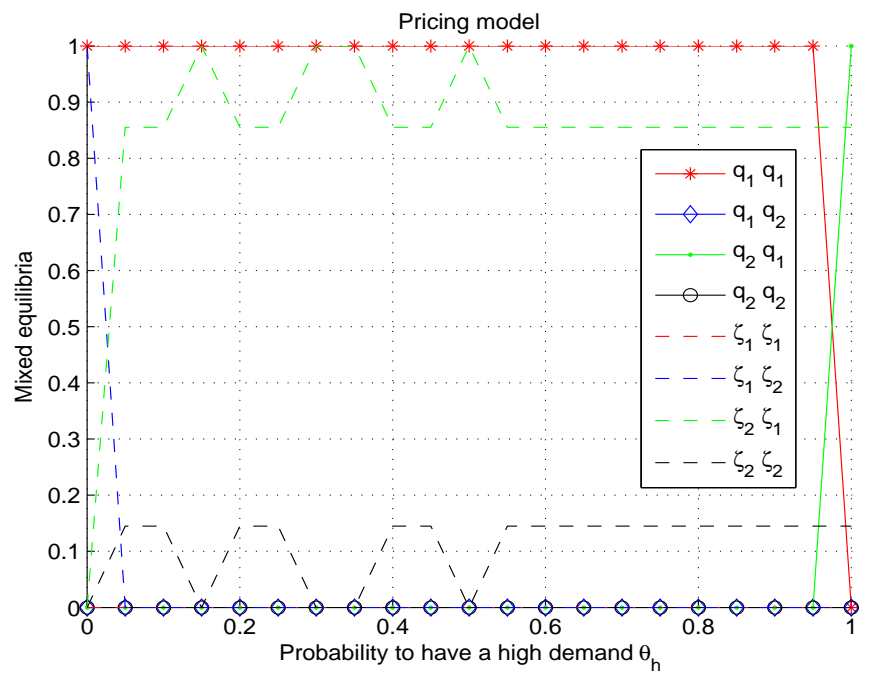

Fig. 4. Service providers' mixed equilibria for the pricing model as function of the probability $\pi_{h}$ to have a high demand $\theta_{h}$ with $q_{l}=1, q_{h}=35, \zeta_{l}=$ $0, \zeta_{h}=10, \theta_{l}=1$ and $\theta_{h}=20$.

are illustrated in Figure 4 using the following values $q_{l}=$ $1, q_{h}=20, \zeta_{l}=0, \zeta_{h}=10, \theta_{l}=1$ and $\theta_{h}=20$. One can be clearly see that the first player almost always chooses the low pricing level policy $q_{1} q_{1}$ regardless the demand it observes. On the other side, in order to maximize its own utility, the second player observes the action of the first player and chooses with probability $\simeq 0.85$ policy $\zeta_{2} \zeta_{1}$ and with probability $\simeq 0.15$ policy $\zeta_{2} \zeta_{2}$ as the probability $\pi_{h}$ increases.

\section{CONCLUSION AND Discussion}

In this paper, we studied a model of pricing access to the Internet and investigated the role of signalling (or recommen- 
dations) in identifying side payments (price per unit demand). We considered the case of two Internet service providers aiming at maximizing their own expected utility. Under this setting, we assume that that the amount of information made available to both ISPs on the demand function is not the same: the more informed provider plays first, and then the second observes the move of the first provider and chooses accordingly its own action.

We studied two configurations of the proposed problem. In the first configuration, one service provider (say provider 1) has a fixed price known to the other provider (say provider 2). The pure actions of provider 1 are thus the possible choices of what signal to send. In the second configuration, provider 1 is allowed to choose his prices which serves as a signal to provider 2 . We then reduced each one of the configuration to an equivalent four by four matrix game and studied the equilibria

Our model is, needless to say, a mere caricature that captures certain types of interactions between ISPs. The biggest benefit is that it is tractable, as evidenced by the obtained expressions in this paper. The litmus test of its usefulness will be its ability, or otherwise, to explain some observed behavior, even if only qualitatively. Studies in this direction are ongoing. Finally, an extension of our model to account for aspects of quality of service (QoS) should be brought in to enrich the model. We hope to pursue some of these in future works.

\section{REFERENCES}

[1] D. M. Kreps and J. Sobel, "Signaling", Chapter 25 in R.J. Aumann and S. Hart (ed.), Handbook of Game Theory with Economic Applications, Elsevier, edition 1, volume 2, pages 849-867, 1994.

[2] D. Braess, "ber ein Paradoxon aus der Verkehrsplanung", Unternehmensforschung, No. 12, pp. 258-268, 1968. 